\title{
C-Band Line of Sight Communication for analysis to Compact Microstrip Patch Antenna Using Parasitic Technique
}

\author{
Dharavat Ravinayak \\ Assistant Professor,Department of Electronics and Communication Engineering, \\ Dadi Institute of Engineering and Technology, Anakapalli, Andhra Pradesh, India \\ E-Mail: ravinayak46@gmail.com
}

\begin{abstract}
The investigation of microstrip fix radio wires has gained awesome ground as of late. Contrasted and ordinary reception apparatuses, microstrip fix recieving wires have more points of interest and better prospects. They are lighter in weight, low volume, ease, low profile, littler in measurement and simplicity of manufacture and similarity. Besides, the microstrip fix reception apparatuses can give double and round polarizations, double recurrence activity, recurrence spryness, wide band-width, feedline adaptability, shaft checking unidirectional designing. In this paper we examine the microstriprecieving wire, kinds of microstrip reception apparatus, sustaining methods and use of microstrip fix radio wire with their favorable position and detriments over ordinary microwave radio wires.From this paper, design of small Microstrip patch antenna using parasitic technique for range of sight communication is proposed, having hexagonal condition ground plane. The recommended antenna has slots in the patch to obtain wideband. The antenna model is simulated using Finite component method at the rate of recurrence 2. $65 \mathrm{GHz}$ to 4 . $57 \mathrm{GHz}$. The antenna design demonstrates desired features for wireless communication

Keywords: Satellite, Microstrip patch antenna, parasitic technique, C-Band, S-Band, Line of Sight Communication
\end{abstract}

\section{INTRODUCTION}

Antenna is a transducer designed to transmit or receive electromagnetic waves. Microstrip antennas have several advantages over conventional microwave antenna and therefore are widely used in many practical applications. Microstrip antennas in its simplest configuration are shown in Fig1. It consists of a radiating patch on one side of dielectric substrate $(\mathrm{Cr} \leq 10)$, which has a ground plane on other side.Microstrip antennas have always been an attractive choice for the researchers, especially in the field where light weight and easy to fabricate structures are desired such as in line of sight communication or Mobile communication. Microstrip Patch antenna is known to be a low profile antenna, which can be mounted on a flat surface. It is basically designed with radiating patch on one side of a dielectric substrate and on the other side of this dielectric substrate, a ground plane is fixed. The patch is

Usually made up of various conducting materials such as copper or gold and can be produced in any possible shape. Electric fringing fields between the edges of the conductor element and the ground plane behind it are the primary source of the antenna's radiation. The antenna's radiation depends upon various properties of antenna such as dielectric constant, height of the substrate, the patch dimensions and the frequency. The radiating patch ismade up of a perfect electric conductor material on one side of its substrate is made up of material Rogers RT/Duroid 5880 and the ground plane on the other side of the substrate[1-2]. These antennas are designed mainly for single mode operation, but as the number of users and number of desired applications in a single device are increasing day by day, demand of wideband antennas is also growing in order to fulfil suchneeds.

\section{BACKGROUND}

\section{A. C-Band}

It is the original frequency allocation for communications satellites. C-Band uses 3.7-4.2GHz for downlink and 5.925$6.425 \mathrm{GHz}$ for uplink. The lower frequencies that $\mathrm{C}$ Band uses perform better under adverse weather conditions than the Kuband or Ka band frequencies.

\section{B. S-Band}

It is part of the electromagnetic spectrum's microwave band, which is defined by the standards set by the IEEE for radio waves. The frequency range for the $S$ band is 2 to $4 \mathrm{GHz}$, which crosses the conventional boundary between UHF and $\mathrm{SHF}$, which is at $3 \mathrm{GHz}$.

\section{Patch antenna}

A patch antenna (also known as a rectangular Microstrip antenna) is a type of radio antenna with a low profile, which can be mounted on a flat surface. It consists of a flat rectangular sheet or "patch" of metal, mounted over a larger sheet of metal called a ground plane.

\section{L-Band}

It is the operating frequency range of $1-2 \mathrm{GHz}$ in the radio spectrum. The wavelength range of $\mathrm{L}$ band is $30-15 \mathrm{~cm}$. The $\mathrm{L}$ band is one of the chief operating ranges used by various applications such as radars, global positioning systems (GPS), radio, and telecommunications and aircraft surveillance. 


\section{EXISTING WORK}

\section{A. Literature work}

A Microstrip patch antenna (MPA) consists of a conducting patch of any planar or nonplanar geometry on one side of a dielectric substrate with a ground plane on other side. It is a popular printed resonant antenna for narrow-band microwave wireless links that require semi hemispherical coverage. Due to its planar configuration and ease of integration with Microstrip technology, the Microstrip patch antenna has been heavily studied and is often used as elements for an array.

A large number of Microstrip patch antennas have been studied to date. An exhaustive list of the geometries along with their salient features is available [1]. The rectangular and circular patches are the basic and most commonly used Microstrip antennas. These patches are used for the simplest and the most demanding applications.

Rectangular geometries are separable in nature and their analysis is also simple. The circular patch antenna has the advantage of their radiation

\section{B. Feeding Techniques}

A feedline is used to excite to radiate by direct or indirect contact. There are many different techniques of feeding and four most popular techniques are coaxial probe feed, Microstrip line, aperture coupling and proximity coupling [2]. Coaxial probe feeding is feeding method in which that the inner conductor of the coaxial is attached to the radiation patch of the antenna while the outer conductor is connected to the ground plane. Advantages of coaxial feeding is easy of fabrication, easy to match, low spurious radiation and its disadvantages is narrow bandwidth, Difficult to model specially for thick substrate.

\section{Applications}

The Microstrip patch antennas are well known for their performance and their robust design, fabrication and their extent usage. The advantages of this Microstrip patch antenna are to overcome their de-merits such as easy to design, light weight etc., the applications are in the various fields such as in the medical applications, satellites and of course even in the military systems just like in the rockets, aircrafts missiles etc. the usage of the Microstrip antennas are spreading widely in all the fields and areas and now they are booming in the commercial aspects due to their low cost of the substrate material and the fabrication.

It is also expected that due to the increasing usage of the patch antennas in the wide range this could take over the usage of the conventional antennas for the maximum applications. Microstrip patch antenna has several applications. Some of these applications are discussed as below: Mobile and satellite communication application:
Mobile communication requires small, low-cost, low profile antennas. Microstrip patch antenna meets all requirements and various types of microstrip antennas have been designed for use in mobile communication systems.

In case of satellite communication circularly polarized radiation patterns are required and can be realized using either square or circular patch with one or two feed points. Global Positioning System applications: Nowadays microstrip patch antennas with substrate having high permittivity sintered material are used for global positioning system.

These antennas are circularly polarized, very compact and quite expensive due to its positioning. It is expected that millions of GPS receivers will be used by the general population for land vehicles, aircraft and maritime vessels to find there position accurately.

\section{Radio Frequency Identification (RFID)}

RFID uses in different areas like mobile communication, logistics, manufacturing, transportation and health care [2]. RFID system generally uses frequencies between $30 \mathrm{~Hz}$ and 5.8 GHz depending on its applications. Basically RFID system is a tag or transponder and a transceiver or reader.

\section{E. Worldwide Interoperability for Microwave Access (WiMAX)}

The IEEE 802.16 standard is known as WiMAX. It can reach upto 30 mile radius theoretically and data rate 70 Mbps. MPA generates three resonant modes at 2.7, 3.3 and $5.3 \mathrm{GHz}$ and can, therefore, be used in WiMAX compliant communication equipment.

\section{F. Radar Application}

Radar can be used for detecting moving targets such as people and vehicles. It demands a low profile, light weight antenna subsystem, the microstrip antennas are an ideal choice. The fabrication technology based on photolithography enables the bulk production of microstrip antenna with repeatable performance at a lower cost in a lesser time frame as compared to the conventional antennas.

\section{G. Rectenna Application}

Rectenna is a rectifying antenna, a special type of antenna that is used to directly convert microwave energy into DC power. Rectenna is a combination of four subsystems i.e. Antenna, ore rectification filter, rectifier, post rectification filter. inrectenna application, it is necessary to design antennas with very high directive characteristics to meet the demands of long-distance links. Since the aim is to use the rectenna to transfer DC power through wireless links for a long distance, this can only be accomplished by increasing the electrical size of the antenna. 


\section{H. Telemedicine Application}

In telemedicine application antenna is operating at 2.45 GHz. Wearable Microstrip antenna is suitable for Wireless Body Area Network (WBAN). The proposed antenna achieved a higher gain and front to back ratio compared to the other antennas, in addition to the semi directional radiation pattern which is preferred over the Omnidirectional pattern to overcome unnecessary radiation to the user's body and satisfies the requirement for on-body and off-body applications. A antenna having gain of $6.7 \mathrm{~dB}$ and a $\mathrm{F} / \mathrm{B}$ ratio of $11.7 \mathrm{~dB}$ and resonates at $2.45 \mathrm{GHz}$ is suitable for telemedicine applications.

\section{Medicinal applications of patch}

It is found that in the treatment of malignant tumors the microwave energy is said to be the most effective way of inducing hyperthermia. The design of the particular radiator which is to be used for this purpose should posses light weight, easy in handling and to be rugged. Only the patch radiator fulfils these requirements.

The initial designs for the Microstrip radiator for inducing hyperthermia was based on the printed dipoles and annular rings which were designed on S-band. And later on the design was based on the circular Microstrip disk at L-band. There is a simple operation that goes on with the instrument; two coupled Microstrip lines are separated with a flexible separation which is used to measure the temperature inside the human body. A flexible patch applicator can be seen in the figure below which operates at $430 \mathrm{MHz}$.

\section{PROPOSED WORK}

\section{A. Antenna Model and Design}

In this paper a compact Microstrip patch antenna is presented using parasitic technique for line of sight communication. It consists of slots in the patch to enhance the bandwidth of the designed model [3-8]. By optimization of the length and width of these slots, wideband is obtained.

Figure.1: shows the geometry of the proposed antenna Which is a coaxial-fed slotted patch for line of sight operation in the wireless communication? The antenna formed by the strip which is directly printed on the patch portion. In this antenna model, the patch has size of $20 \mathrm{~mm}$ $\mathrm{X} 24 \mathrm{~mm}$ and substrate is made from a $1.6 \mathrm{~mm}$ thick RT/Duroid 5880 substrate of relative permittivity 2.2 and loss tangent is 0.0009 . The ground plane used here is of hexagonal shape to improve the radiation pattern of the antenna.

Microstrip antenna design equations as mentioned in equations (1-4) have been used to calculate width, length and effective dielectric constant for proposed antenna.

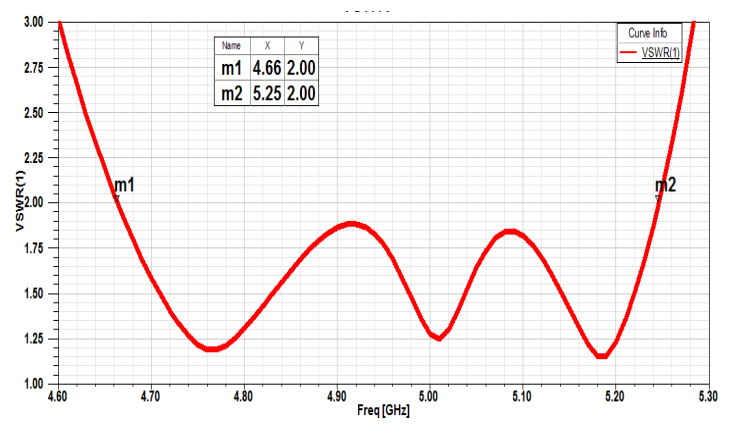

Fig. 1 Geometry of the proposed antenna

$$
\begin{gathered}
W=\frac{c}{2 f_{r}}\left(\frac{\varepsilon_{r}+1}{2}\right)^{-\frac{1}{2}} \\
L=\frac{c}{2 f_{r} \sqrt{\varepsilon_{e}}}-2 \Delta l \\
\varepsilon_{e}=\frac{\varepsilon_{r}+1}{2}+\frac{\varepsilon_{r}-1}{2}\left(1+\frac{12 h}{W}\right)^{-\frac{1}{2}} \\
\frac{\Delta l}{h}=0.412 \frac{\left(\varepsilon_{e}+0.3\right)\left(\frac{W}{h}+0.264\right)}{\left(\varepsilon_{e}-0.258\right)\left(\frac{W}{h}+0.8\right)}
\end{gathered}
$$

where the following parameters are used, $f_{r}$ is the resonant frequency, w is the width of the patch, $\mathrm{L}$ is the length of the Patch, $\mathrm{h}$ is the thickness of substrate, $\varepsilon_{\mathrm{r}}$ is the relative permittivity of the dielectric substrate, $\varepsilon_{\mathrm{e}}$ is effective dielectric constant and $\mathrm{c}$ is speed of light

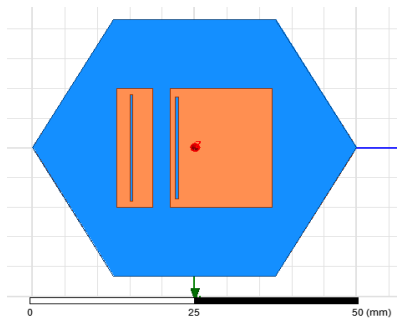

Fig. 2Proposed antenna model

\section{V.SIMULATED RESULTS}

After proper modeling of the proposed antenna it has been simulated and optimized using HFSS software. Simulated results of the antenna is shown in Fig. II (a, b, c and d). Fig. 2 shows that simulated VSWR of the antenna is less than 2 throughout the band i.e. $4.66 \mathrm{GHz}$ to $5.25 \mathrm{GHz}$ and Fig. 3-5 shows the radiation pattern. Fig. 6 and 7 shows the gain versus frequency and directivity versus frequency respectively.

From the Fig. it is very clear that at the desired frequency band of operation the gain is greater than $6 \mathrm{dBi}$, in between there is a slight change which is due the parasitic technique but this does not make any affect in the performance of theantenna. 


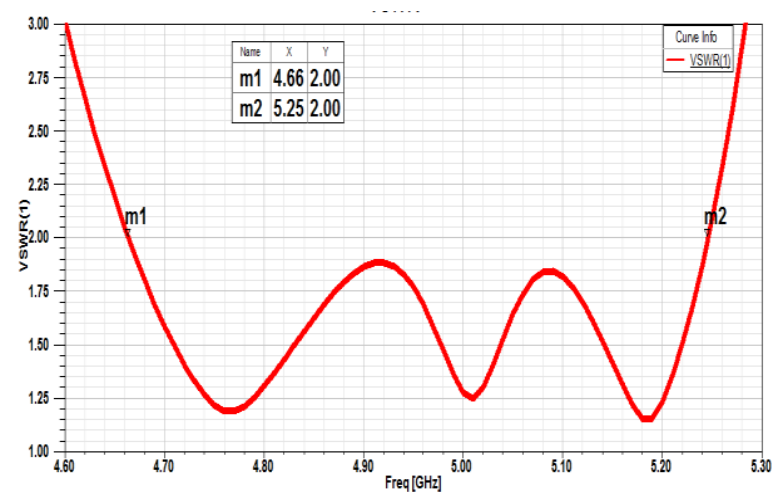

Fig. 3Variation of VSWR v/s Frequency for proposed antenna

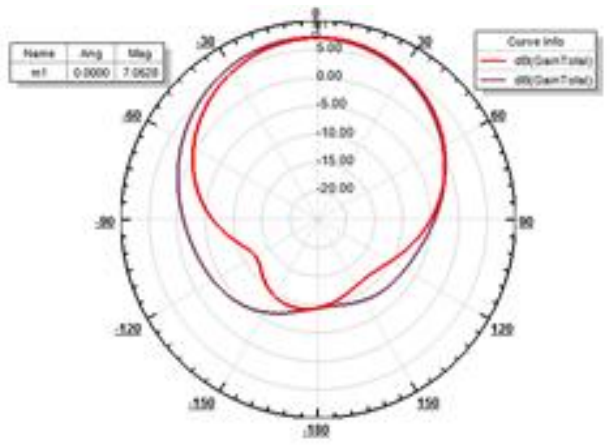

Fig. 4 Radiation pattern of proposed antenna at $4.7 \mathrm{GHz}$

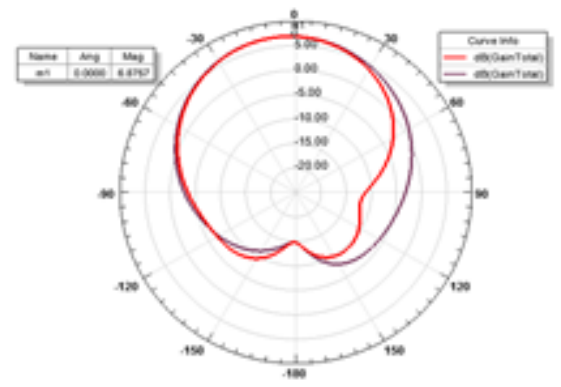

Fig.5 Radiation pattern of proposed antenna at $4.8 \mathrm{GHz}$

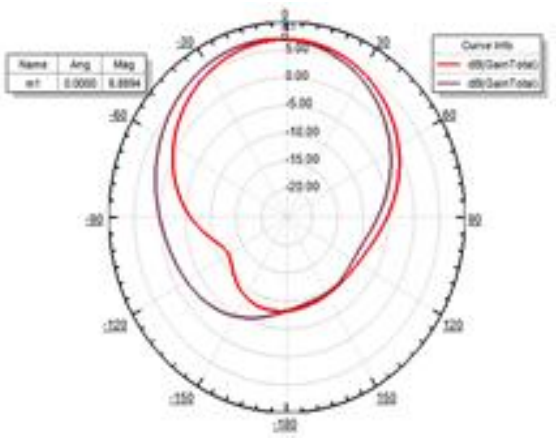

Fig. 6 Radiation pattern of proposed antenna at $5.2 \mathrm{GHz}$

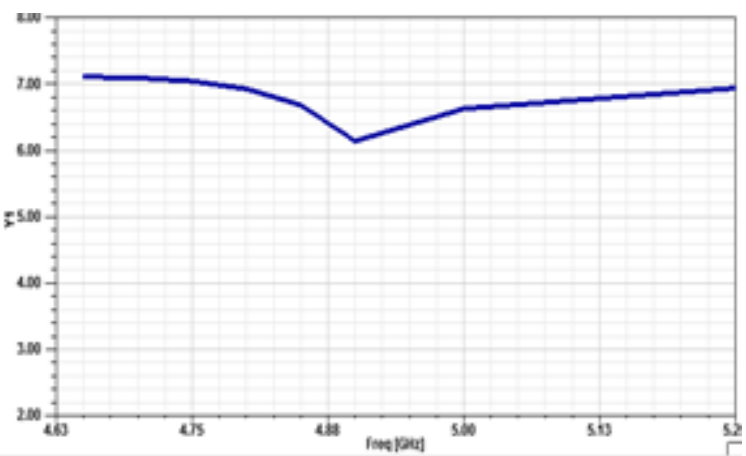

Fig. 7 Radiation pattern of proposed antenna at $5.2 \mathrm{GHz}$

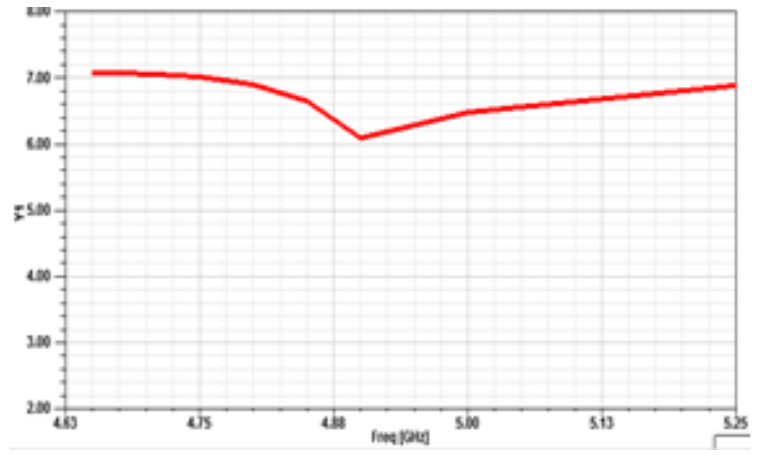

Fig. 7 Variation of directivity v/s frequency for Proposed antenna

\section{CONCLUSION}

A low profile innovative compact Microstrip patch antenna using parasitic technique has been designed and simulated using HFSS software. Impedance bandwidth of the order of $12.89 \%$ has been achieved in single layer Microstrip configuration. Simulated gain of the designed antenna is more than $6 \mathrm{dBi}$ over the band of operation. The designed antenna can be used in line of sight communication system at C-Band.

\section{REFERENCES}

[1] Constantine A Balanis, Antenna Theory Analysis and Design, Wiley student edition, 2008 .

[2] I.J.Bahl and P.Bhartia, Microstrip Antennas Design Handbook, Dedham, MA: Artech House, 1981.

[3] R.S.Kushwaha, D.K.Srivastava, S. Dhupkariya, J.P saini and K. Prasad, "Slot loaded Electromagnetically Coupled Microstrip Line Fed Microstrip Patch Antenna for Wideband Applications",IEEE Conference on Communications and Signal Processing (ICCSP), 1082-1086, 2013.

[4] R.Caso, A. A. Serra, M.Pino, P.Nepa and G.Manara, "A Wideband Slot-Coupled Stacked-Patch Array for Wireless Communications", IEEE Antennas Wireless Propagation Letters, Vol. 9, pp.986-989, 2010.

[5] Mohammad Tariqul Islam, Norbahiah Misran, BaharudinYatim, Analysis of Broadband Microstrip Patch Antenna, IEEE Proceedings on ICCIT, pp. 758-761, 2008. 